\title{
Interface shape control using localized heating during Bridgman growth
}

\author{
M. P. Volz ${ }^{\mathrm{a}, *}$, K. Mazuruk ${ }^{\mathrm{b}}$, M. D. Aggarwal $^{\mathrm{c}}$, A. Cröll $^{\mathrm{d}}$ \\ ${ }^{a}$ NASA Marshall Space Flight Center, EM30, Huntsville, AL 35812, USA \\ ${ }^{b}$ University of Alabama in Huntsville, Huntsville, AL 35899, USA \\ ${ }^{c}$ Department of Physics, Alabama A\&M University, Huntsville, AL 35762, USA \\ ${ }^{d}$ Kristallographisches Institut,University of Freiburg, Hebelstr. 25, D-79104 Freiburg, Germany \\ *Corresponding author. Tel. 256 544-5078; Fax. 256 544-8762; email: Martin.Volz@nasa.gov
}

\begin{abstract}
Numerical calculations were performed to assess the effect of localized radial heating on the melt-crystal interface shape during vertical Bridgman growth. System parameters examined include the ampoule, melt and crystal thermal conductivities, the magnitude and width of localized heating, and the latent heat of crystallization. Concave interface shapes, typical of semiconductor systems, could be flattened or made convex with localized heating. Although localized heating caused shallower thermal gradients ahead of the interface, the magnitude of the localized heating required for convexity was less than that which resulted in a thermal inversion ahead of the interface. A convex interface shape was most readily achieved with ampoules of lower thermal conductivity. Increasing melt convection tended to flatten the interface, but the amount of radial heating required to achieve a convex interface was essentially independent of the convection intensity.
\end{abstract}

PACS: 81.10.-h; 81.10.Fq

Keywords: A1. Computer simulation; A1. Convection; A1. Heat transfer; A1. Interfaces; B2. Bridgman technique 


\section{Introduction}

The quality of crystals grown by vertical Bridgman-type techniques depend on the shape of the melt-crystal interface [1]. If the interface is concave with respect to the growing crystal, defects generated at the interface near the ampoule wall can propagate inward. Thermal stresses, resulting from large interface curvatures, can also lead to the generation of defects. In general, a desired goal is to obtain an interface that is slightly convex so that both the propagation of defects from the ampoule wall and thermal stresses are minimized.

Heat transfer determines the isotherms in a Bridgman system, and the melt-crystal interface is set by the isotherm at the melting-point. Heat transfer in the system near the interface can be considered to arise from two primary factors [2]. The first is the penetration of radial temperature variations from the hot and cold zones into the gradient zone. A typical Bridgman system consists of a hot zone, gradient zone, and cold zone. Changes in the axial temperature gradient at the transition between zones lead to radial temperature gradients. The second primary factor affecting heat transfer at the melt-crystal interface has been termed the "interface effect" $[3,4]$. The interface effect is due to differences in the thermal conductivity of the melt and crystal phases of material directionally solidified in ampoule container. The difference in thermal conductivity between melt and crystal leads to a change in the axial temperature gradient at the interface in the material but the change in axial gradient within the adjacent ampoule is continuous. This results in heat transfer between the solidifying material and the ampoule and a nonplanar interface. Latent heat released at the interface during solidification can also be a significant contributor to the interface effect. If the thermal conductivity of the melt is greater than the crystal, such as is the case for many semiconductor systems, than the ampoule will be cooler than the crystal and the negative radial temperature gradient will cause a concave interface 
shape. If the thermal conductivity of the melt is less than the crystal, as is true for some metallic systems and oxides, than a convex interface shape will result.

There have been several papers which have considered methods to control the meltcrystal interface shape [1-14]. Jasinski et al. [2] analyzed how the position of the melt-crystal interface within the furnace gradient zone can affect its shape. For semiconductor systems, if the interface is positioned near the top end of the gradient zone near the hot zone, radial variations in the temperature will cause the macroscopic interface shape to be convex. Conversely, the interface shape will be concave if positioned near the bottom of the gradient zone. Brandon and Derby [9] analyzed the growth of oxide crystals, for which the thermal conductivity is higher in the solid than the liquid, and found that the interface could be made flatter (less convex) by moving it toward the cold end of the gradient region. Hofman et al. [10] were able to grow Ge crystals with a flat or slightly convex interface shape using a vertical gradient freeze process. This was accomplished by positioning the interface in a shallow axial temperature gradient with a very steep drop off in temperature below it in the solid. Lun et al. [14] analyzed the growth of cadmium zinc telluride in a Bridgman-type furnace. They considered a range of applied axial thermal profiles and a perturbation to the profile below the melting point. If the axial thermal gradient below the melting point was increased, the interface could be made less concave. However, over the range of parameters studied, adjustments to the furnace profile were not able to reverse the interface shape.

Heat transfer by radiation can also affect the interface shape. For nonopaque crystals, Brandon and Derby [9] found that radial thermal gradients were dramatically altered from the interaction of internal radiation and ampoule heat transfer and led to large deflections of the melt-crystal interface. They were able to decrease the interface curvature by using low emissivity 
inner ampoule walls. Radiation properties were used to advantage by Szofran and Lehoczky [7]. They were able to flatten the interface for part of the growth of HgCdTe by adjusting the radiation view angles from the interface to the hot and cold zones of the furnace.

The above methods to control the interface shape do not directly counteract the interface effect. They can, under certain conditions, be used to develop an interface shape that is convex in the center of the crystal but that may still be concave at the crystal-ampoule boundary [4]. The ideal way to counteract the interface effect is to provide an infinitesimally narrow radial inward flow of heat at the melt-crystal interface. One method to try and achieve this is the application of local heating to the ampoule exterior at the melt-crystal interface. The effect of local heating on the interface shape was previously analyzed by Jasinski and Witt [4] using a finite-element thermal model. They applied local heating with a width $40 \%$ of the sample radius and centered at the growth interface. They considered the case of Ge and CdTe grown in boron nitride ampoules. For smaller amounts of surface heating, the interface was convex in the middle but remained concave near the ampoule wall. The entire interface became convex as the surface heating was increased. Lan et al. [13] used a booster heater at the interface during the horizontal Bridgman growth of sodium nitrate. They obtained a convex interface shape which significantly improved the yield of single crystals.

In this study, we present a numerical analysis of the effect that local surface heating can have on the shape of the melt-crystal interface. The local heating is applied in the center of the furnace gradient zone. The gradient region is long enough that such that changes in the thermal gradients at the transition between furnace zones do not affect the melt-crystal interface. The effects of several system parameters are presented. These include the magnitude and width of the 
localized heating, the ampoule, melt and crystal thermal conductivities, and the latent heat of crystallization. The effect of heat transfer by convection in the melt is also evaluated.

\section{Model formulation}

A schematic diagram of the model Bridgman crystal growth system is shown in Fig. 1a. A cylindrical ampoule with radius $r_{0}$ and height $h$ is filled with a solidifying melt. A characteristic temperature profile, applied to the ampoule exterior and translated with velocity $v_{\mathrm{g}}$ with respect to the ampoule, is shown in Fig. 1b. It consists of a hot zone with temperature $t_{\mathrm{H}}$, a cold zone with temperature $t_{\mathrm{C}}$, and a gradient zone with length $l_{\mathrm{G}}$. Additional local heating is applied in the linear gradient region, centered on the crystal-melt interface.

Several modeling assumptions have been made in order to focus on the control of interface morphology by additional localized heating at the interface. The system is in a quasi-

steady-state and transients are neglected. The thermal properties of the ampoule, melt and crystal are not temperature dependent. Heat transfer is dominated by conduction and convection. Only axially symmetric solutions are considered.

The computational domain consists of three regions: the melt, crystal, and ampoule. The heat and mass transfer are solved in cylindrical coordinates using the Boussinesq approximation and the vorticity formulation of the Navier-Stokes equations. The vorticity is given as $\boldsymbol{\omega}=(\nabla \times \mathbf{v}) \varliminf_{\varphi}$,

where $v$ is the velocity of flow in the melt. Dimensionless governing equations can be written as

$$
\begin{aligned}
& \nabla^{2} \Omega-\frac{\Omega}{R^{2}}+\frac{V_{R}}{R} \Omega-\frac{d \Omega}{d z} V_{Z}-\frac{d \Omega}{d R} V_{R}+G r \frac{d T}{d R}=0 \\
& K \nabla^{2} T=\operatorname{Pr}\left(V_{Z} \frac{d T}{d Z}+V_{R} \frac{d T}{d R}\right)
\end{aligned}
$$


$\nabla^{2} V_{R}=\frac{d \Omega}{d Z}+\frac{V_{R}}{R^{2}}$

$\nabla^{2} V_{Z}=-\frac{d \Omega}{d R}-\frac{\Omega}{R}$

where $\Omega$ is the vorticity, $V_{R}$ and $V_{Z}$ are the $R$ and $Z$ components of the melt velocity, $T$ is the temperature, $K$ is the thermal conductivity, $G r$ is the Grashof number and $P r$ is the Prandtl number of the melt. In the above equations, capitalized variables indicate that they are dimensionless and the distance, velocity, vorticity, and thermal conductivity are scaled by $r_{0}, \frac{v}{r_{0}}, \frac{v}{r_{0}^{2}}$, and $k_{\mathrm{m}}$, respectively. Here $v$ and $k_{\mathrm{m}}$ are the viscosity and thermal conductivity of the melt. The dimensionless temperature $T$ is scaled according to $T=\frac{t-t_{C}}{t_{H}-t_{C}}$

The Grashof and Prandtl numbers are given by

$G r=\frac{\beta g r_{0}^{3}\left(t_{H}-t_{C}\right)}{v^{2}}, \quad \operatorname{Pr}=\frac{v}{\kappa}$,

where $\beta$ is the thermal expansion coefficient, $g$ is the gravitational acceleration, and $\kappa$ is the melt thermal diffusivity.

Boundary conditions are required for the variables $T, V_{R}, V_{Z}$, and $\Omega$. The temperature is continuous across all regions. In addition, the temperature is defined along the exterior of the ampoule wall as

$T=T_{\mathrm{C}}$, $Z<H_{i}-\frac{L_{G}}{2}$

$T=\left(Z-H_{i}+\left(\frac{L_{G}}{2}\right)\right) G_{T}+T_{p} \exp \left(-4 \ln (2)\left(\frac{Z-H_{i}}{T_{f w h m}}\right)^{2}\right), \quad H_{i}-\frac{L_{G}}{2}<Z<H_{i}+\frac{L_{G}}{2}$ 
$T=T_{\mathrm{H}}$,

$$
H_{i}+\frac{L_{G}}{2}<Z,
$$

where $H_{i}$ is the vertical position of the crystal-melt interface at the ampoule wall and $G_{T}$ is the applied temperature gradient. We assume that the additional local heating has a Gaussian shape with the magnitude and width of the local heating given as $T_{p}$ and $T_{f w h m}$. Heat flux conditions at the crystal-ampoule and melt-ampoule interfaces are given as

$$
K_{c}\left(\frac{\partial T}{\partial R}\right)_{c}=K_{a}\left(\frac{\partial T}{\partial R}\right)_{a}, \quad K_{m}\left(\frac{\partial T}{\partial R}\right)_{m}=K_{a}\left(\frac{\partial T}{\partial R}\right)_{a},
$$

where $K_{c}$ and $K_{a}$ are the thermal conductivities of the crystal and ampoule. At the crystal-melt interface,

$$
K_{m}\left(\frac{\partial T}{\partial n}\right)_{m}=V_{g} \Delta H \rho+K_{c}\left(\frac{\partial T}{\partial n}\right)_{c}
$$

where $\Delta H \rho$ is the energy per volume of latent heat released during crystallization.

No-slip boundary conditions are specified for the melt velocity on the interior ampoule surfaces and along the crystal-melt interface. Axisymmetry is enforced through the following conditions along the cylinder axis:

$V_{R}=0, \quad \frac{\partial V_{Z}}{\partial R}=0, \quad$ for $R=0$

The vorticity boundary conditions are specified as follows:

$$
\begin{array}{ll}
\Omega=0 & \text { for } R=0 \\
\Omega=\frac{\partial V_{R}}{\partial Z}, & \text { for } Z=H, \\
\Omega=-\frac{\partial V_{Z}}{\partial R}, & \text { for } R=1 \\
\Omega=\frac{\partial V_{R}}{\partial Z}, & \text { at the crystal-melt interface. }
\end{array}
$$


The shape of the crystal-melt interface is not specified prior to the calculations, but is determined by the heat flux conditions (Eqs. 11-12) and by the constraint that along the interface $T=0.5$. The commercial software FlexPDE 5.0 was used in the calculations without fluid flow. The same model was formulated and calculated with the commercial software COMSOL Multiphysics 3.4. Several identical cases were run with both sets of software and gave the same results to within the desired accuracy. COMSOL Multiphysics was used in those cases where melt convection was included. Calculations were performed using a limited number of parameter values representative of semiconductor crystal growth in vertical Bridgman configurations. The ratio of crystal to melt thermal conductivity was set to 0.5 , which is reasonable for liquid semiconductor systems [15]. The ratios of the ampoule height and wall thickness to the ampoule radius were set to 12 and 0.1 , respectively.

\section{Results and discussion}

A Gaussian temperature perturbation is applied in the center of the furnace gradient region and on the exterior of the ampoule. The applied temperature profiles for four different values of $T_{\mathrm{p}}$ are shown at the bottom of Fig. 2 . The temperature perturbations have a full width at half maximum of 2. Close-up views of the resulting isotherms near the crystal-melt interface are shown at the top of the figure. The height of the contour plots is $2 R$. The ampoule is on the right of the plots and the centerline of the melt is on the left. The interface near the center of the plots has a value of $T=0.5$ and the other isotherms are evenly spaced at $\Delta T=0.04$. In these plots the ratio of the ampoule to melt thermal conductivity is 0.05 , which is comparable to Ge grown in fused silica. For $T_{\mathrm{p}}=0$, the interface is concave with respect to the crystal, as expected in systems where the thermal conductivity of the melt is greater than that of the crystal [4]. As $T_{\mathrm{p}}$ 
increases, the interface flattens and then becomes convex. The additional localized heating tends to counteract the cooling effect of the ampoule. At the crystal-melt interface, the temperature inside the ampoule increases from the interior to the exterior for $T_{\mathrm{p}}=0$ but decreases for $T_{\mathrm{p}}=0.3$. The applied temperature is nonmonotonic at the ampoule exterior for $T_{\mathrm{p}}=0.3$, but as a result of thermal diffusion, becomes monotonic along the interior wall of the ampoule.

The amount of interface deflection depends on the ratio of ampoule to melt thermal conductivity. The interface deflection versus $T_{\mathrm{p}}$ is shown in Fig. 3 for three different values of $K_{\mathrm{a}}$. The interface deflection is concave above the solid line at $Z=0$, and convex below it. It is clear that additional local heating can reverse the interface shape, even for widely varying values of ampoule thermal conductivity. Also, the strength of $T_{\mathrm{p}}$ required to reverse the deflection is somewhat independent of $K_{\mathrm{a}}$. Ampoules of lower thermal conductivity do tend to flatten the interface, whether the interface is concave or convex.

When the interface is made convex as a result of local radial heating, the condition $\frac{\partial T}{\partial R}>0$ is not necessarily met along the entire interface. Fig. 4 shows the interface shapes for three values of $K_{\mathrm{a}}$ when $T_{\mathrm{p}}=0.2$. Although the interface is found at larger $Z$ in the center of the ampoule than along the edge for all values of $K_{\mathrm{a}}$, the interface is concave near the ampoule wall for $K_{\mathrm{a}}=5$. As $K_{\mathrm{a}}$ increases, the radial thermal gradient within the ampoule decreases, and local heating is less able to affect the interface curvature at the melt-crystal-ampoule tri-junction.

Local radial heating does lower the vertical temperature gradient at the crystal-melt interface. This can be seen in the contour plots of Fig. 2, where the vertical distance between isotherms becomes larger as $T_{\mathrm{p}}$ increases. Fig. 5 shows the minimum value of $\frac{\partial T}{\partial Z}$ at the interface as a function of $T_{\mathrm{p}}$. The effect of local radial heating puts a practical upper limit on $T_{\mathrm{p}}$. 
A smaller axial temperature gradient necessitates a smaller growth velocity in order to avoid constitutional supercooling. But as shown in Fig. 3, the transition between concave and convex occurs when $0.1<T_{\mathrm{p}}<0.15$, where the minimum value of $\frac{\partial T}{\partial Z}$ is still quite large. The effect of $T_{\mathrm{p}}$ on $\frac{\partial T}{\partial Z}$ can be further mitigated by using ampoules of lower thermal conductivity. Such ampoules can tolerate a larger amount of radial heating before the axial temperature gradient becomes negative.

For a given value of $T_{\mathrm{p}}$, a convex interface shape can be more readily achieved with a narrower radial heat perturbation. Shown in Fig. 6 is the interface deflection versus $T_{\mathrm{p}}$ for three values of the FWHM. Also shown are representative temperature profiles applied at the ampoule exterior for $T_{\mathrm{p}}=0.2$. Clearly, a narrower temperature perturbation will more readily balance the thermal conductivity mismatch at the tri-junction. However, from a practical standpoint, it may be more difficult to achieve a narrower perturbation as well as maintain its position at the crystalmelt interface during growth.

Fig. 7 is a plot of the interface deflection versus the dimensionless latent heat of crystallization released at the interface. The additional heat energy released at the interface does increase the interface deflection and increases the concavity of an already concave interface. The solid horizontal line at 0 demarcates the transition from convex to concave. The vertical arrow in the lower left-hand corner of the plot indicates the position of Ge grown at $1.4 \mu \mathrm{m} / \mathrm{s}$. Ge has a relatively large heat of fusion and it is possible to achieve a convex interface shape in that system for the given conditions. Growth at significantly higher velocities or the growth of material with a higher latent heat of crystallization would require greater radial heating to maintain a convex interface. 
The effect of local radial heating on convection in the melt is shown in Fig. 8. The data for all four plots were generated with the following parameters: $G r=10^{7}, \operatorname{Pr}=0.05, K_{\mathrm{a}}=0.05$, and $T_{\mathrm{fwhm}}=2$. Plots a, b, c, and d used the values of $T_{\mathrm{p}}=0.0 .1,0.2$, and 0.3 respectively. When $T_{\mathrm{p}}=0, \frac{\partial T}{\partial R}<0$ at the interface, and the radial temperature gradient drives a clockwise flow cell near the interface. When $T_{\mathrm{p}}=0.1$, the interface is nearly flat and no significant flow exists. As $T_{\mathrm{p}}$ increases, the interface becomes more convex. Because $\frac{\partial T}{\partial R}>0$ at the interface, a counterclockwise flow cell develops. The presence of convection has only a minimal impact on the ability of radial heating to affect the interface shape. Fig. 9 shows the interface deflection versus $T_{\mathrm{p}}$, both with and without melt convection. The presence of strong convection does not significantly affect the strength of local heating required to change from a concave to a convex interface shape. For both concave and convex interfaces, the presence of convection serves to simply reduce the interface curvature.

\section{Summary}

Application of local radial heating at the crystal-melt interface is a relatively simple way to reverse the interface shape from concave to convex. The required strength and spatial extent of additional heating can readily be achieved with modern multizone furnaces under computer control. The strength of local heating required to reverse the interface shape is relatively independent of ampoule thermal conductivity, but a lower ampoule thermal conductivity will result in a smaller decrease in the axial temperature gradient at the interface. A narrower heat perturbation requires less additional heating than a broader one to achieve a convex interface shape. The latent heat of fusion does require a larger value of local heating to reverse the 
interface shape, but for typical parameters used in semiconductor crystal growth, this is readily achievable. Increased melt flow (i.e., larger Grashof number) tends to flatten the interface, whether concave or convex, but cannot reverse its shape and does not significantly change to strength of additional local heating required to reverse the interface shape.

\section{Acknowledgement}

Authors M. P. Volz and M. D. Aggarwal are grateful for the support received from the NASA Administrators Fellowship Program during this work.

\section{References}

1. C. E. Chang, W. R. Wilcox, J. Crystal Growth 21 (1974) 135.

2. T. Jasinski, A. F. Witt, W. M. Rohsenow, J. Crystal Growth 67 (1984) 173.

3. R. J. Naumann, S. L. Lehoczky, J. Crystal Growth 61 (1983) 707.

4. T. Jasinski, A. F. Witt, J. Crystal Growth 71 (1985) 295.

5. $\quad$ S. Sen, W. R. Wilcox, J. Crystal Growth 28 (1975) 36.

6. T-W. Fu, W. R. Wicox, J. Crystal Growth 48 (1980) 416.

7. $\quad$ F. R. Szofran, S. L. Lehoczky, J. Crystal Growth 70 (1984) 349.

8. S. Sen, W. H. Konkel, S. J. Tighe, L. G. Bland, S. R. Sharma, R. E. Taylor, J. Crystal Growth 86 (1988) 111.

9. $\quad$ S. Brandon, J. J. Derby, J. Crystal Growth 121 (1992) 473.

10. D. Hofmann, T. Jung, G. Müller, J. Crystal Growth 128 (1993) 213.

11. P. S. Dutta, K. S. Sangunni, H. L. Bhat, V. Kumar, J. Crystal Growth 141 (1994) 44.

12. P. S. Dutta, H. L. Bhat, V. Kumar, J. Crystal Growth 154 (1995) 213. 
13. C. W. Lan, M. C. Su, M. C. Liang, J. Crystal Growth 208 (2000) 717.

14. L. Lun, A. Yeckel, M. Reed, C. Szeles, P. Daoutidis, J. J. Derby, J. Crystal Growth 290 (2006) 35.

15. A. S. Jordan, J. Crystal Growth 71 (1985) 551.

\section{Figure Captions}

Fig. 1. Schematic diagram of the (a) Bridgman crystal growth system and (b) the temperature profile with additional local heating.

Fig. 2. Applied temperature profiles and resulting isotherms for $K_{\mathrm{a}}=0.05, T_{\mathrm{fwhm}}=2$, and for $T_{\mathrm{p}}=0.1,0.2,0.3$ and 0.4

Fig. 3. Interface deflection versus $T_{\mathrm{p}}$ for three values of the ampoule to melt thermal conductivity ratio $K_{\mathrm{a}}$.

Fig. 4. Melt-crystal interface shapes for $K_{\mathrm{a}}=0.05,0.5$ and 5 . The strength and width of the local heating are $T_{\mathrm{p}}=0.2$ and $T_{\mathrm{fwhm}}=2$.

Fig. 5. Minimum value of the axial temperature gradient at the interface versus $T_{\mathrm{p}}$.

Fig. 6. Interface deflection versus $T_{\mathrm{p}}$ for $T_{\mathrm{fwhm}}=1,2$, and 3 .

Fig. 7. Interface deflection versus the dimensionless latent heat of crystallization released at the interface. The latent heat released by Ge grown at $5 \mathrm{~mm} / \mathrm{hr}=1.4 \mu \mathrm{m} / \mathrm{s}$ is shown.

Fig. 8. Isotherms and flow vectors resulting from additional local heating: (a) $T_{\mathrm{p}}=0$, (b) $T_{\mathrm{p}}=0.1$, (c) $T_{\mathrm{p}}=0.2$, (c) $T_{\mathrm{p}}=0.3$.

Fig. 9. Interface deflection versus $T_{\mathrm{p}}$ for $G r=0$ and $G r=10^{7}$. 


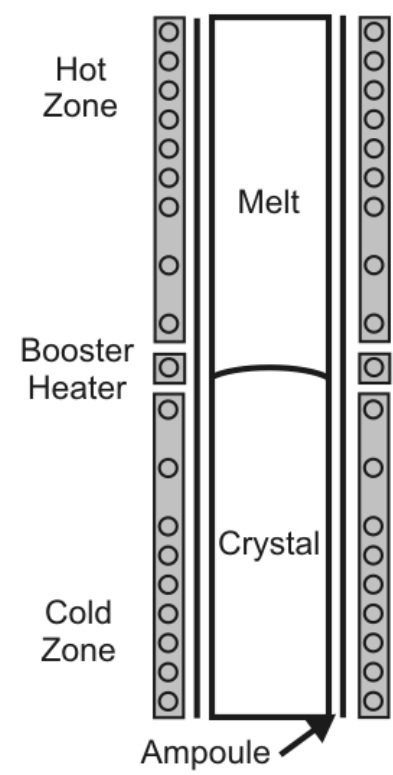

(a)

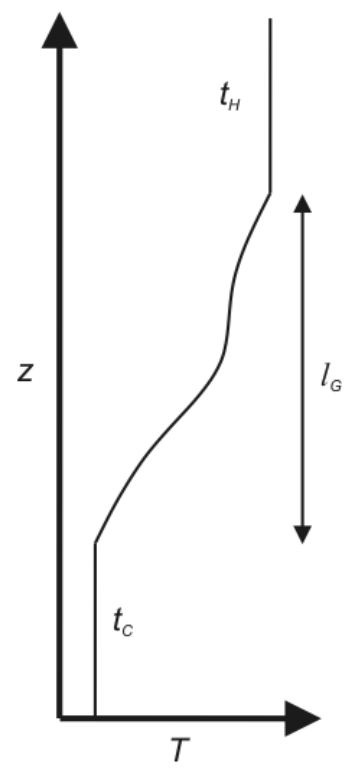

(b)

Fig. 1. Schematic diagram of the (a) Bridgman crystal growth system and (b) the temperature profile with additional local heating.
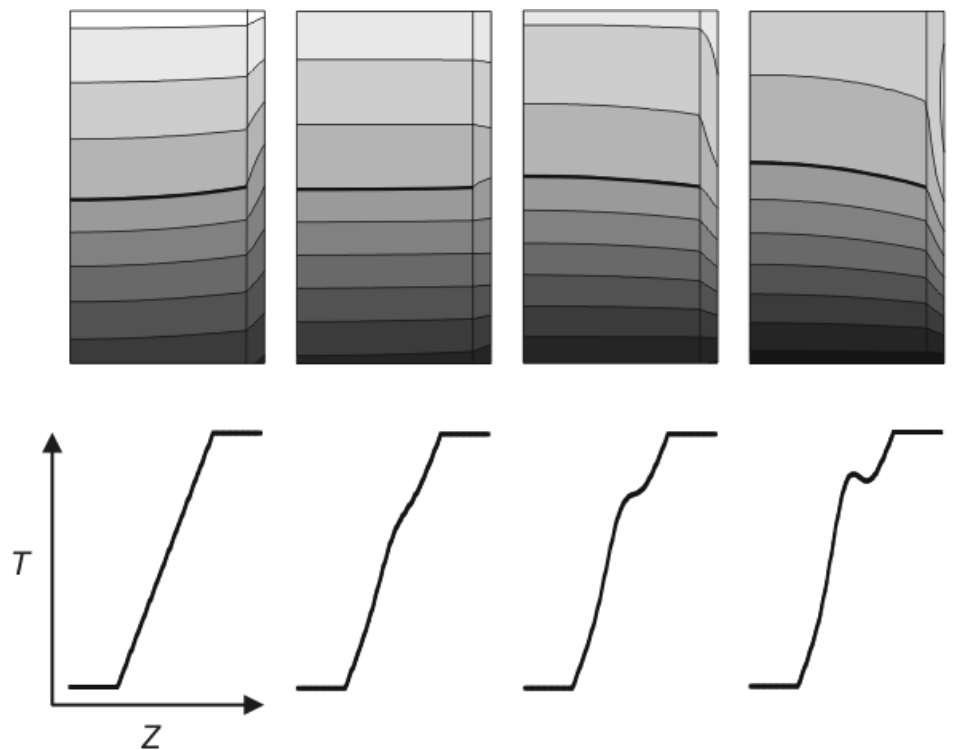

$T_{p}=0$

$T_{\rho}=0.1$

$T_{\mathrm{p}}=0.2$

$T_{\rho}=0.3$

Fig. 2. Applied temperature profiles and resulting isotherms for $K_{\mathrm{a}}=0.05, T_{\mathrm{fwhm}}=2$, and for $T_{\mathrm{p}}=0.1,0.2,0.3$ and 0.4 . 


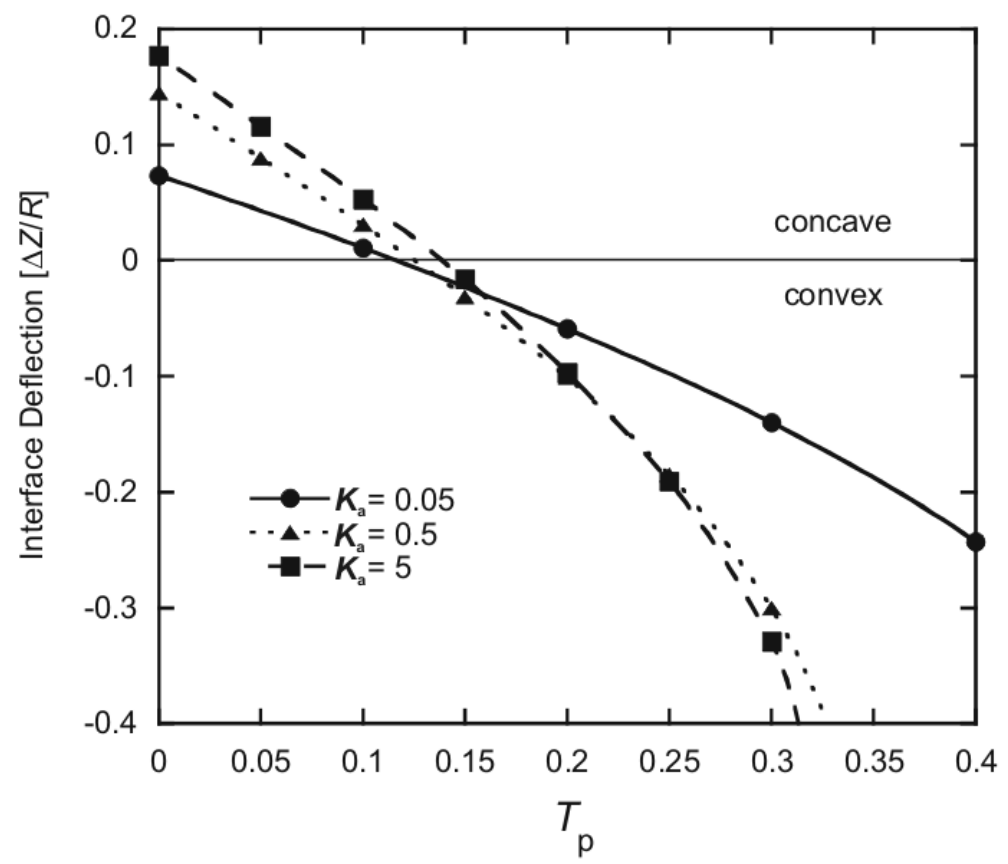

Fig. 3. Interface deflection versus $T_{\mathrm{p}}$ for three values of the ampoule to melt thermal conductivity ratio $K_{\mathrm{a}}$.

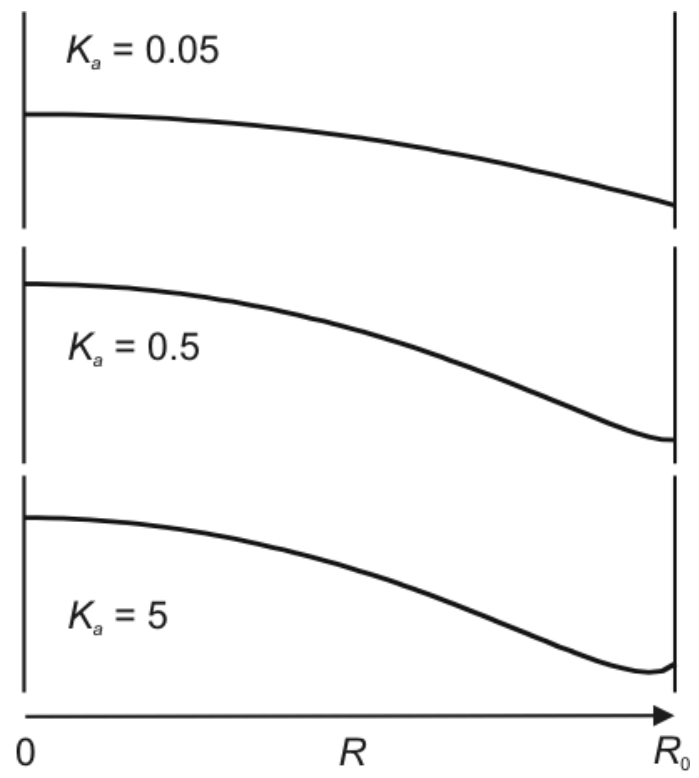

Fig. 4. Melt-crystal interface shapes for $K_{\mathrm{a}}=0.05,0.5$ and 5. The strength and width of the local heating are $T_{\mathrm{p}}=0.2$ and $T_{\mathrm{fwhm}}=2$. 


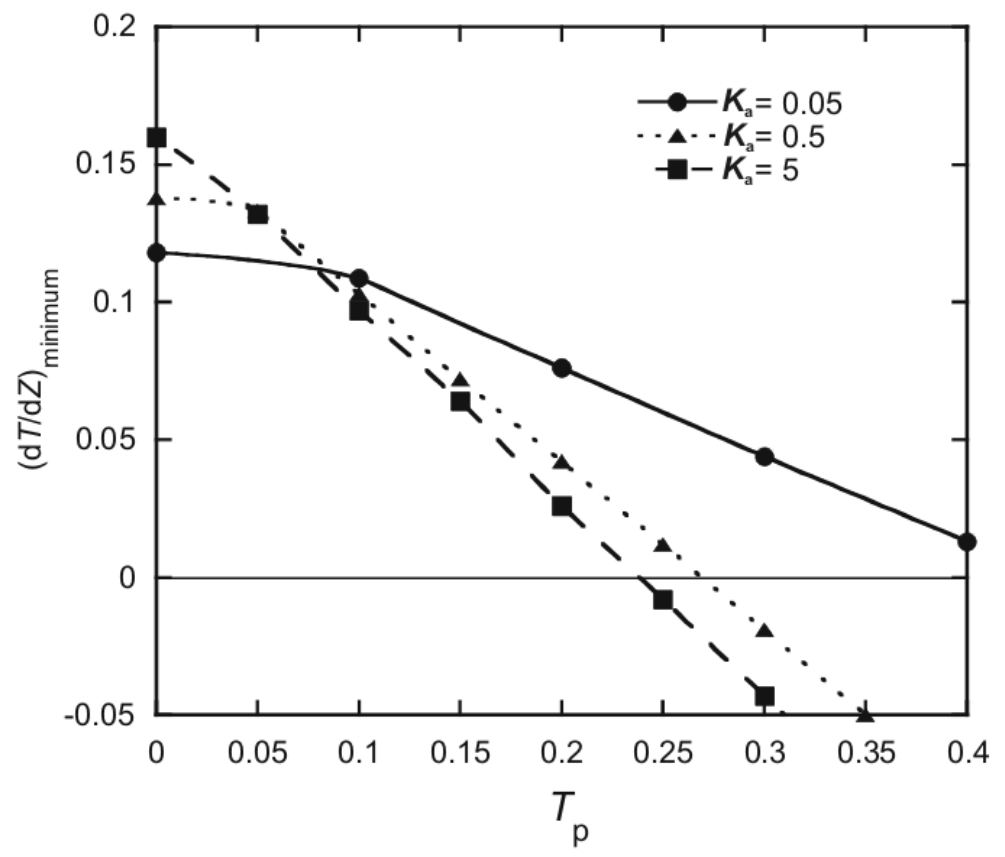

Fig. 5. Minimum value of the axial temperature gradient at the interface versus $T_{\mathrm{p}}$.

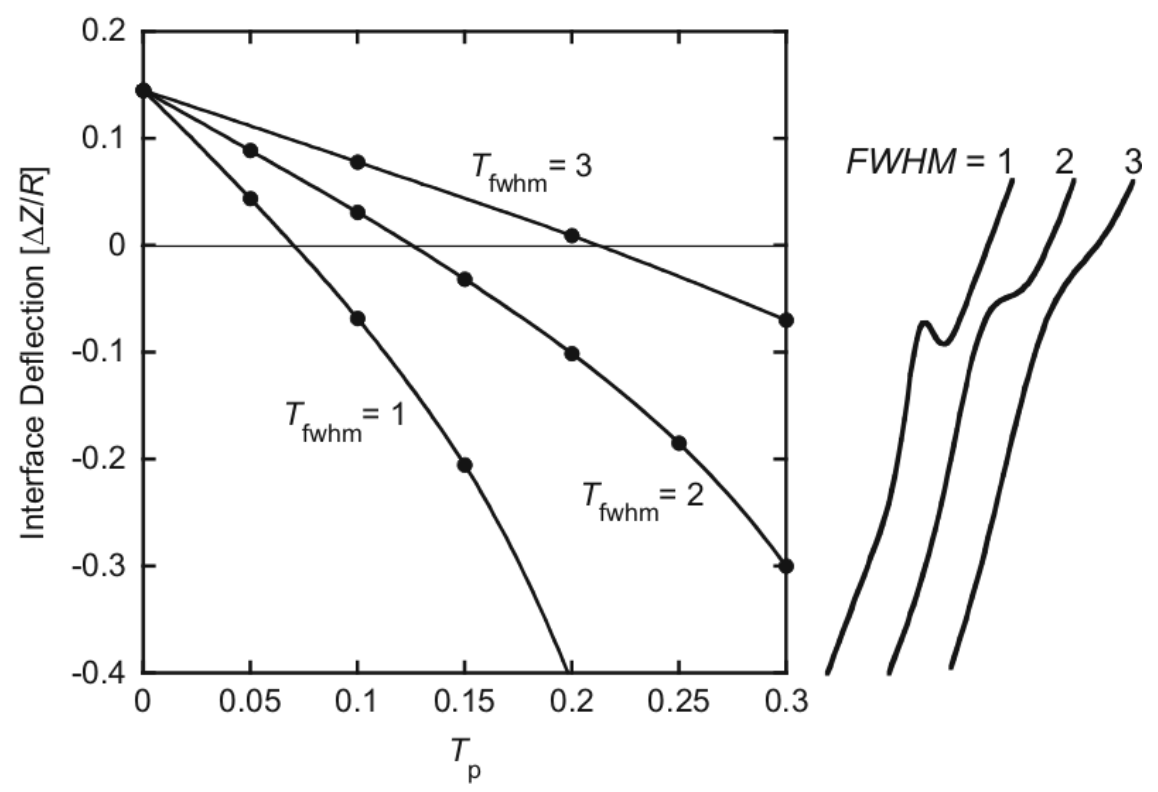

Fig. 6. Interface deflection versus $T_{\mathrm{p}}$ for $T_{\mathrm{fwhm}}=1,2$, and 3 . 


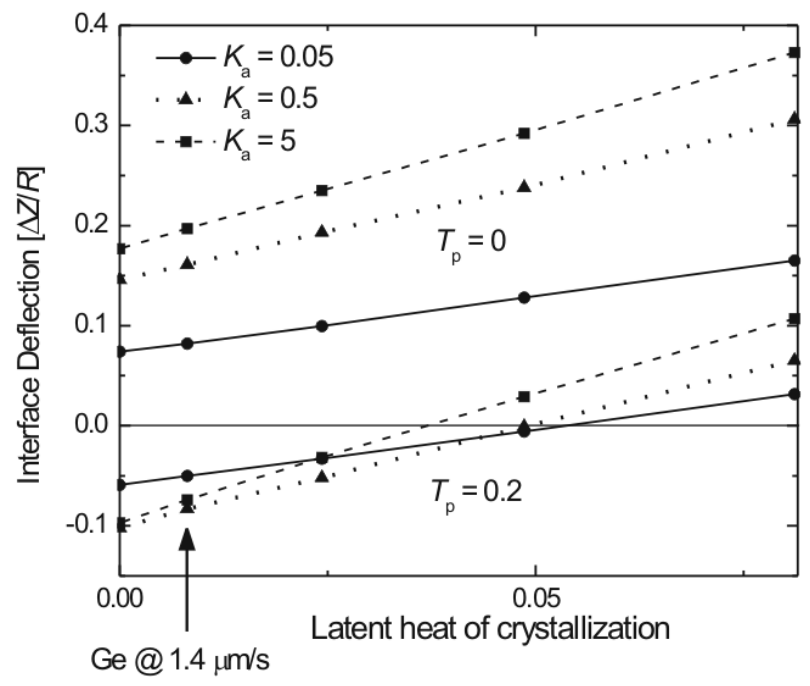

Fig. 7. Interface deflection versus the dimensionless latent heat of crystallization released at the interface. The latent heat released by Ge grown at $5 \mathrm{~mm} / \mathrm{hr}=1.4 \mu \mathrm{m} / \mathrm{s}$ is shown.

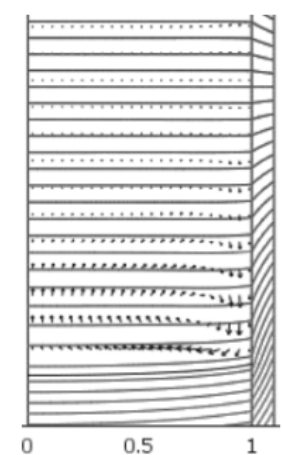

(a)

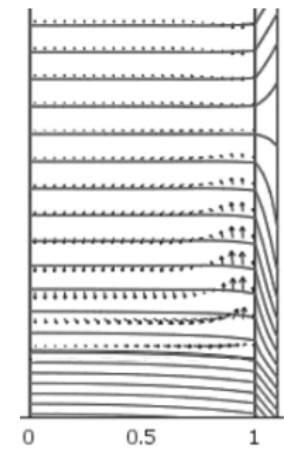

(c)

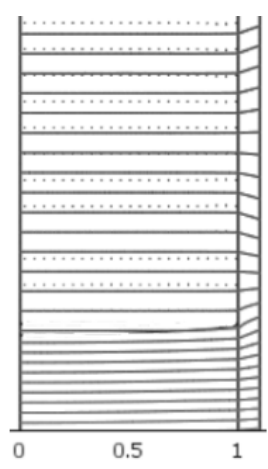

(b)

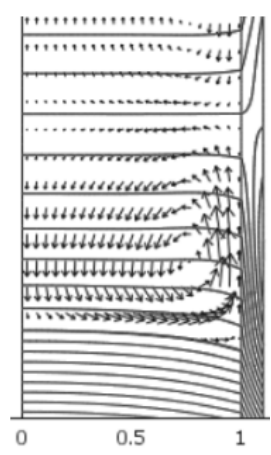

(d)

Fig. 8. Isotherms and flow vectors resulting from additional local heating: (a) $T_{\mathrm{p}}=0$,

(b) $T_{\mathrm{p}}=0.1$, (c) $T_{\mathrm{p}}=0.2$, (c) $T_{\mathrm{p}}=0.3$. 


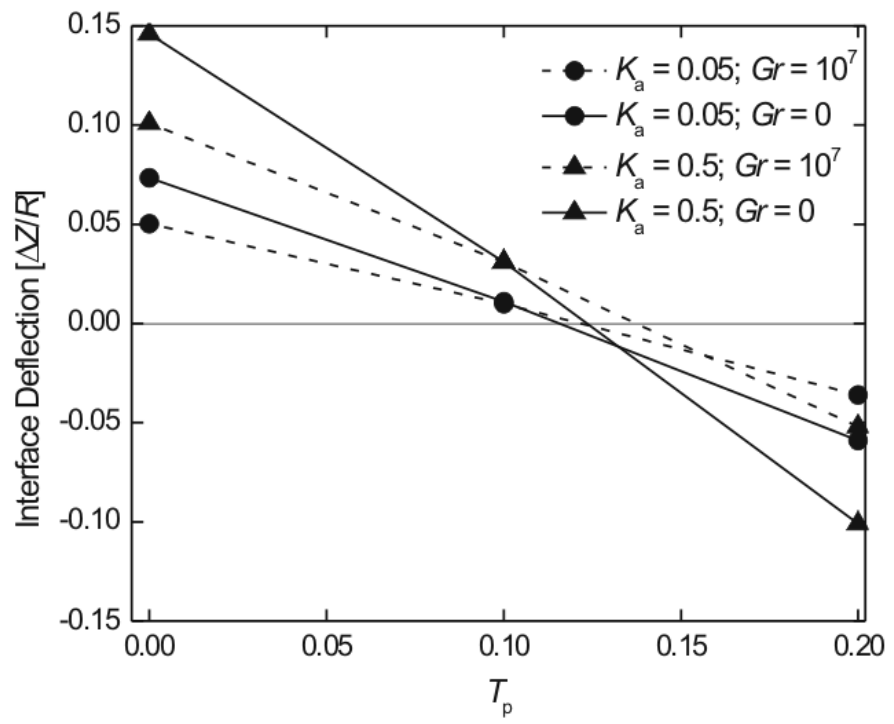

Fig. 9. Interface deflection versus $T_{\mathrm{p}}$ for $G r=0$ and $G r=10^{7}$. 